\title{
Research lab on 3D bioprinting of Zhejiang University
}

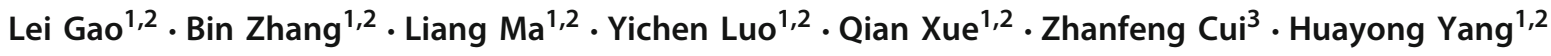

Received: 21 May 2018 / Accepted: 23 May 2018 / Published online: 4 June 2018

(c) Zhejiang University Press 2018

\section{Overview}

The Research Lab on 3D Bioprinting of Zhejiang University focuses on developing innovative 3D bioprinting equipment and technology for healthcare and biomedical applications, since founded in 2017. To further enhance the international collaboration, a joint international research lab on 3D bioprinting of Zhejiang University and University of Oxford was set up. The lab is based on the State Key Laboratory of Fluid Power and Mechatronic Systems and the Oxford Centre for Tissue Engineering and Bioprocessing. The aim of the lab is to establish a world class research and development platform on 3D bioprinting. The lab has extensive and close collaborations with University of Oxford, University College London and multidisciplinary partners of Zhejiang University.

Currently, the lab focuses on the development of highresolution 3D bioprinters with multi-materials printability and the applications of 3D bioprinting technology in engineering tissues and organs. In particular, the lab has developed high-resolution 3D bioprinters with multiple printheads, with the positioning accuracy up to $1 \mu \mathrm{m}$ with high viability and functionality of the printed cells and tissues.

\section{Research staff}

The lab is led by Professor Huayong Yang, Academician, Chinese Academy of Engineering in collaboration with Professor Zhanfeng Cui, fellow, Royal academy of Engineering from University of Oxford.

Professor Huayong Yang, Principle Investigator, Head, School of Mechanical Engineering, Zhejiang University

Director, State Key Laboratory of Fluid Power and Mechatronic Systems, Zhejiang University

Professor Zhanfeng Cui, is a visiting professor in Zhejiang University.

Dr. Bin Zhang and Dr. Liang Ma are in charge of the management of the lab. Dr. Hua Ye, associate professor in University of Oxford, involves some collaborative research projects. Currently, there are 8 Ph.D. students and 8 Master students in the lab.
Huayong Yang

yhy@zju.edu.cn

1 State Key Laboratory of Fluid Power and Mechatronic Systems, Zhejiang University, Hangzhou, China

2 School of Mechanical Engineering, Zhejiang University, Hangzhou, China

3 Department of Engineering Science, Institute of Biomedical Engineering, University of Oxford, Oxford OX3 7DQ, UK 


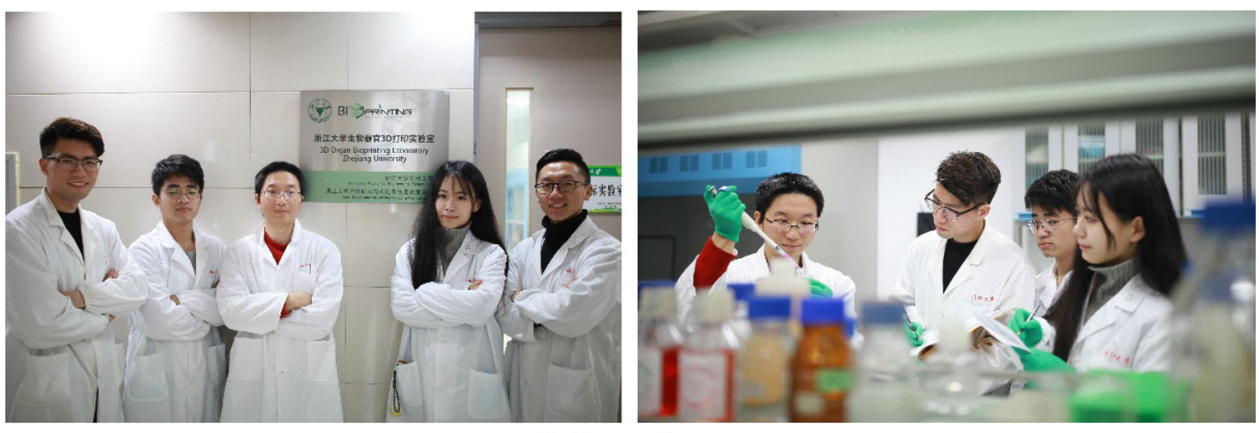

Lab members

\section{Research directions}

The research directions of the lab are as the following:

- The development of 3D bioprinting systems with high precision and multiple DoF (degrees of freedom).

- Vascularized skin tissue printing and the diagnosis of skin diseases based on deep learning.

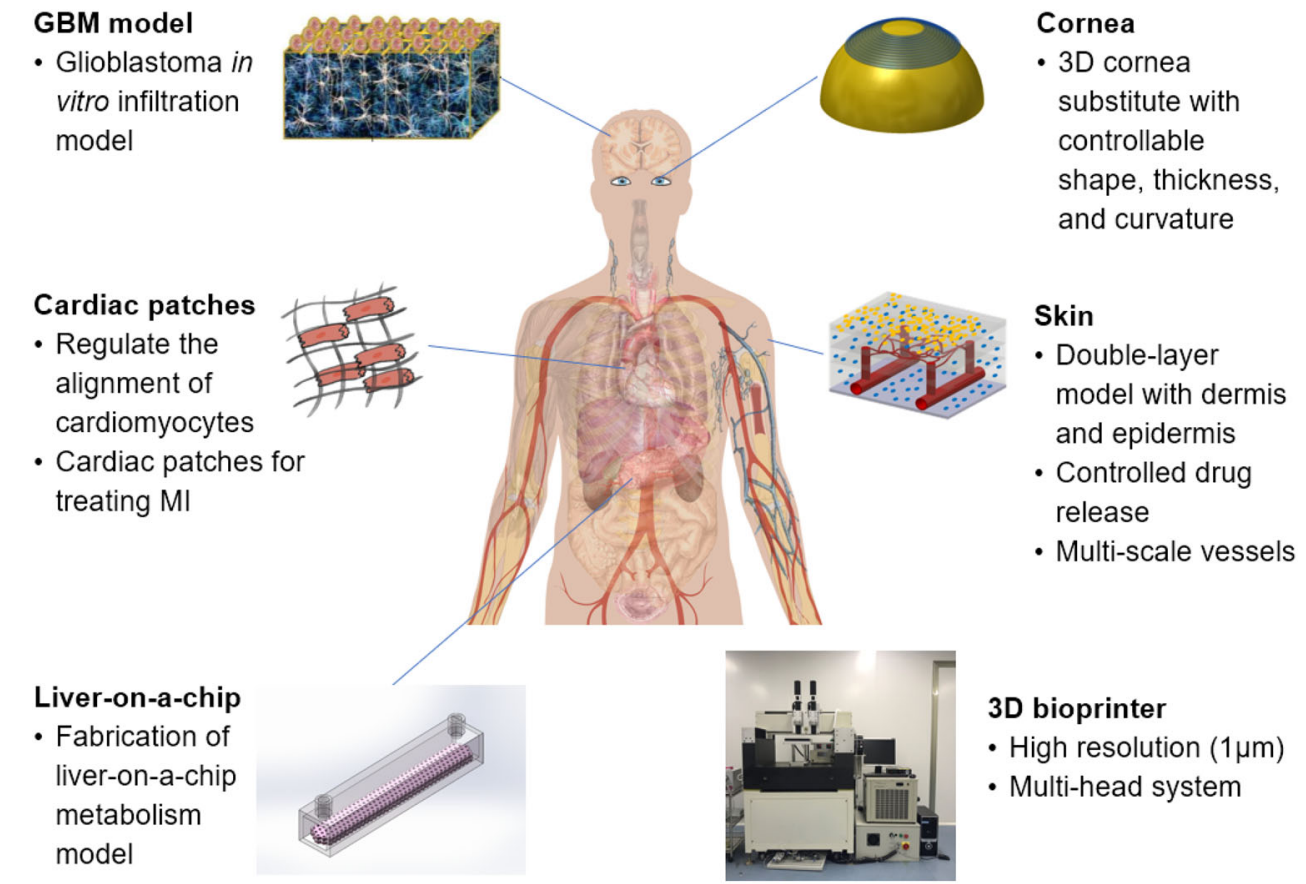

Research directions of the lab
- The study of 3D bioprinting systems and method for corneal substitution with complex surface.

- Control the alignment of cardiomyocytes and construct cardiac patch by 3D bioprinting.

- The construction of tumor in vitro chip model and complex tissues and organs with integrated 3D bioprinting. 


\section{Facilities}

The lab possesses $50 \mathrm{~m}^{2}$ standard 10,000 level clean room for cell culture and cell bioprinting. It is equipped with several advanced facilities including stereo fluorescence microscope for "bioink" preparation, post-printing characterization, and immunofluorescence analysis.
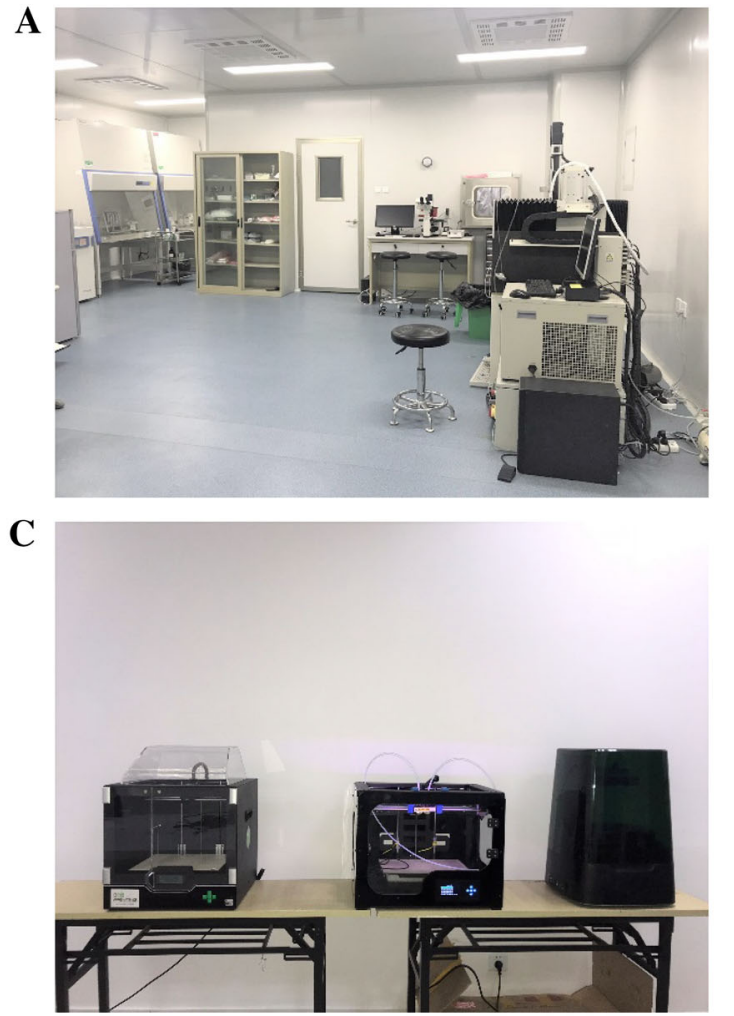

\section{Recent event}

The lab will hold Hangzhou International Conference on Biomaterials, Bio-Design and Manufacturing (BDMC 2018) in conjunction with H2020 BAMOS Project 2018 Meeting at Hangzhou, China, during August 26-28, 2018. BDMC 2018

B

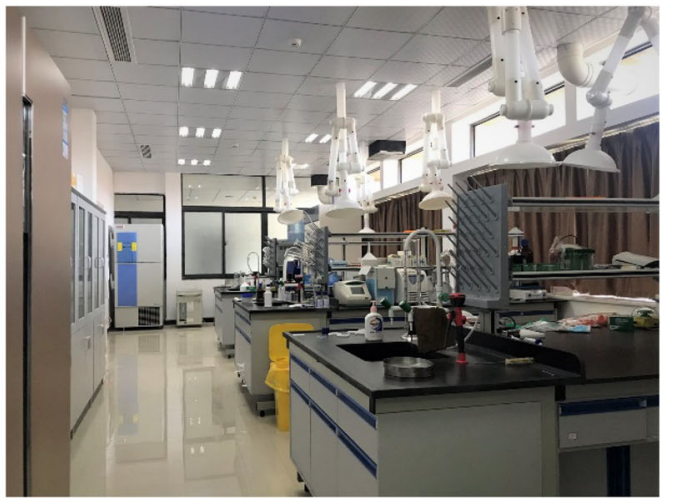

D

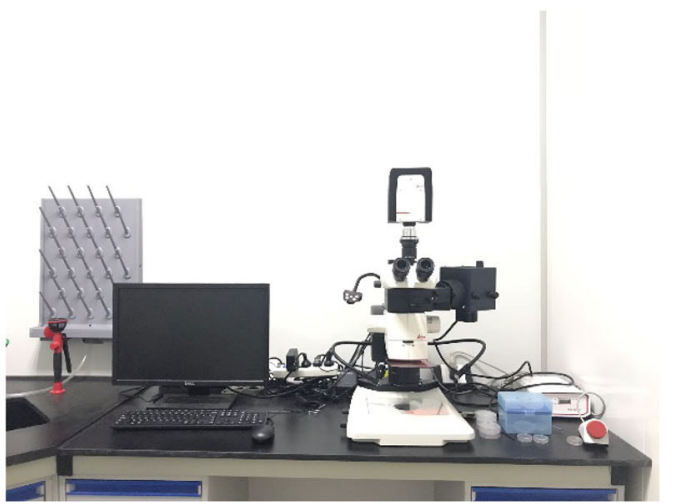

Lab facilities. A. 10000 level clean room. B. Biochemistry lab.

C. 3D printers. D. stereo fluorescence microscope

\section{Perspectives}

Although 3D bioprinting technology has been improved remarkably in aspects of printing speed, precision, reproducibility, etc., it is still in its early stage. Novel formulations of "bioinks" are to be developed to keep the balance between cytocompatibility and printability. The vascularization is also a major challenge for the longevity and long-term functioning of printed tissues and organs. Nevertheless, we envision that $3 \mathrm{D}$ bioprinting will significantly speed up studies on tissue and organ engineering, as well as drug screening and development. will be Co-chaired by Prof. Huayong Yang and Prof. Zhanfeng Cui. More than 200 researchers in this area all over the world will attend this conference. The conference will highlight the following topics:

Topic 1: Novel additive manufacturing mechatronic systems, in particular, 3D bioprinting platforms

Topic 2: Biomaterials and formulation of novel "bioinks" for tissue-specific applications

Topic 3: Engineering tissues and organs in vitro and in vivo 
Topic 4: Design and fabrication of organ-on-a-chip, disease models, bioreactors, and medical and diagnostic devices

Topic 5: Design of therapeutic products, cellular products, and macromolecular products

Special session: BAMOS (Biomaterials and Additive Manufacturing: Osteochondral Scaffold innovation, H2020-MSCA-RISE-2016-734156)

We warmly welcome you to participate in BDMC 2018!

\section{References}

1. Zhang B, Luo Y, Ma L, Gao L, Li Y, Xue Q et al (2018) 3D bioprinting: an emerging technology full of opportunities and challenges. Bio-Des Manuf 1:2-13

2. Yu M, Gong J, Zhou Y, Dong L, Lin Y, Ma L et al (2017) Surface hydroxyl groups regulate the osteogenic differentiation of mesenchymal stem cells on titanium and tantalum metals. J Mater Chem B 5:3955-3963
3. Pei X, Ma L, Zhang B, Sun J, Sun Y, Fan Y et al (2017) Creating hierarchical porosity hydroxyapatite scaffolds with osteoinduction by three-dimensional printing and microwave sintering. Biofabrication 9:045008

4. Yu M, You D, Zhuang J, Lin S, Dong L, Ma L et al (2017) Controlled release of naringin in metal-organic framework-loaded mineralized collagen coating to simultaneously enhance osseointegration and antibacterial activity. ACS Appl Mater Interfaces 9:19698-19705

5. Liu C, Zhou Y, Sun M, Li Q, Dong L, Ma Let al (2017) Light-induced cell alignment and harvest for anisotropic cell sheet technology. ACS Appl Mater Interfaces 9:36513-36524

6. Ma L, Barker J, Zhou C, Li W, Zhang J, Lin B et al (2012) Towards personalized medicine with a three-dimensional microscale perfusion-based two-chamber tissue model system. Biomaterials 33:4353-4361

7. Zhang B, Gao L, Gu L, Yang H, Luo Y, Ma L (2017) High-resolution 3D bioprinting system for fabricating cell-laden hydrogel scaffolds with high cellular activities. Procedia CIRP 65:219-224 